\title{
VLADINOL F, NEOLIGNAN COMPOUND FROM THE STEM BARK OF Dryobalanops oblongifolia (DIPTEROCARPACEAE) AND ANTIPLASMODIAL ACTIVITY
}

\author{
Indriani $^{1}$, N.S. Aminah ${ }^{2,3, \bowtie}$, N.N. Tri Puspaningsih ${ }^{2}$, I.H. Hasna ${ }^{4}$ \\ Y. Takaya ${ }^{5}$ and P. Satrimafitrah ${ }^{1}$ \\ ${ }^{1}$ Department of Chemistry, Tadulako University, Jalan Soekarno Hatta Tondo 94118, Palu, \\ Indonesia. \\ ${ }^{2}$ Department of Chemistry, Faculty of Science and Technology, Universitas Airlangga, \\ Komplek Kampus C, Jl. Mulyorejo, Surabaya, Indonesia. 60115 \\ ${ }^{3}$ Biotechnology of Tropical Medicinal Plants Research Group, Universitas Airlangga \\ ${ }^{4}$ Faculty of Medicine, Universitas Airlangga, Kampus A UNAIR - Jl. Mayjen Prof. Dr. \\ Moestopo 47, Surabaya-60131, Indonesia \\ ${ }^{5}$ Faculty of Pharmacy, Meijo University, 468-8503 Tempaku Nagoya, Japan. \\ ${ }^{{ }}$Corresponding Author: nanik-s-a@fst.unair.ac.id
}

\begin{abstract}
Drybalanops oblongifolia known as kayu kapur is a plant member of Dipterocarpaceae family. A neolignan, vladinol F, was successfully isolated from the dissolved fraction in acetone-ether of Dryobalanops oblongifolia stem bark. The chemical structure's determination was done based on an analysis of UV-Vis, NMR, and MS spectra and a comparison with the reference. In vitro test of this compound against Plasmodium falciparum 3D7 showed that $\mathrm{IC}_{50}$ value was $3.51 \mu \mathrm{g} / \mathrm{mL}$.

Keywords: Drybalanops oblongifolia, Neolignan, Vladinol F, Antiplasmodial Activity, Plasmodium falciparum RASĀYAN J. Chem., Vol. 14, No.1, 2021
\end{abstract}

\section{INTRODUCTION}

Various researches to find new drug candidates have been carried out. ${ }^{1-3}$ Malaria is a parasitic infectious disease which causes millions of deaths every year. Besides that, the resistance of $P$. falciparum to nearly all available antimalarial drugs are increasing today. ${ }^{4}$ Thus, the new antimalarial drugs are urgently needed that will be used to prevent the spread of the infection and to cure infected patients. Dryobalanops oblongifolia is a species of the Dryobalanops genus belonging to the Dipterocarpaceae family. Dryobalanops is called Kayu Kapur by the local community ${ }^{5,6}$ and is recognized as a source of oligostilbene agents. ${ }^{7-10}$ A previously isolated constituent from $D$. oblongifolia reported that dimerstilbene, (-) ampelopsin $\mathrm{F}$, had a potential activity as antiplasmodium. ${ }^{11}$

In continuation of our research program on biologically active compounds from plants, we investigated this plant's antiplasmodial constituent. From this research, we have isolated vladinol $\mathrm{F}$ from the acetone extract of $D$. oblongifolia stem bark.

\section{Material and Methods}

\section{EXPERIMENTAL}

The stem bark of D. oblongifolia was gathered from Gunung Mali, Tempunak, Sintang, West Kalimantan in 2012. The plant was identified at the Biological Research Center, LIPI, Bogor, Indonesia, and the specimen's voucher was stored at the herbarium.

\section{General Procedure}

Using acetone, as many as $5 \mathrm{~kg}$ of D. oblongifolia stem bark powder was macerated twice at room temperature and then concentrated by vacuum evaporator. The acetone extract was divided into two fractions, that were soluble or insoluble in acetone - ether. By vacuum liquid chromatography (VLC) ( $n$-hexane-EtOAc, rising polarity), $48 \mathrm{~g}$ dissolved part in acetone-ether was fractionated to yield fractions A-D. $1.2 \mathrm{~g}$ of fraction D was repeatedly GCC separated using $n$-heksane - EtOAc 5:5 EtOAc $100 \%$ to give subfractions D1 - D3. Subfraction D3 (50 mg), on repeated separation and 
purification using radial chromatography (gradient system of $\left.\mathrm{CHCl}_{3}-\mathrm{MeOH}\right)$, acquired vladinol $\mathrm{F}(10$ mg) (Fig.-1).

\section{Detection Method}

The elucidation structure of the vladinol $\mathrm{F}$ molecule using UV-Vis, ${ }^{1} \mathrm{H}$ and ${ }^{13} \mathrm{C}$ NMR and 2D NMR spectroscopy. ESI-MS spectrometer was used to record the molecular mass of this compound.

\section{RESULTS AND DISCUSSION}

Vladinol F, a colorless amorphous solid, mp. $105^{0}-107^{0}$; UV $\lambda_{\max }(\mathrm{MeOH}): 216,230$, dan $284 \mathrm{~nm}$. ESI-MS: $m / z$ [M-H] ${ }^{-359,2}$ (Fig.-2, supplementary data). ${ }^{1} \mathrm{H}-\mathrm{NMR}$ (methanol-d4, $500 \mathrm{MHz}$ ) (Fig.-1, supplementary data), $\delta_{\mathrm{H}}(\mathrm{ppm}): 1.87(2 \mathrm{H}, m, \mathrm{H}-8 \mathrm{a}) ; 2.63(2 \mathrm{H}, t, \mathrm{H}-7 \mathrm{a}) ; 3.46(1 \mathrm{H}, q, \mathrm{H}-8) ; 3.57(2 \mathrm{H}, t$, $\mathrm{H}-9 \mathrm{a}) ; 3.75(2 \mathrm{H}, d d \& d d$, H-9); $3.81(3 \mathrm{H}, s) ; 3.85(3 \mathrm{H}, s) ; 6.72(1 \mathrm{H}, s, \mathrm{H}-2 \mathrm{a}) ; 6.73(1 \mathrm{H}, s, \mathrm{H}-6 \mathrm{a}) ; 6.76$ $(1 \mathrm{H}, d, \mathrm{H}-3) ; 6.82(1 \mathrm{H}, d d, \mathrm{H}-2) ; 6.95(1 \mathrm{H}, d, \mathrm{H}-6) .{ }^{13} \mathrm{C}-\mathrm{NMR}$ (methanol-d4, $\left.125 \mathrm{MHz}\right)$ (Fig.-3, Suplementary data), $\delta_{\mathrm{C}}(\mathrm{ppm}): 32.9$ (C-7a); $35.8(\mathrm{C}-8 \mathrm{a}) ; 55.5(\mathrm{C}-8) ; 56.4\left(\mathrm{C} 5 \mathrm{a}-\mathrm{OCH}_{3}\right) ; 56.8(\mathrm{C} 5-$ $\left.\mathrm{OCH}_{3}\right) ; 62.2$ (C-9a); 65.0 (C-9); 88.9 (C-7); 110.6 (C-6); 129.9 (C-3a); 114.2 (C-6a); 115.9 (C-3); 117.9 (C-2a); 119.7 (C-2); 134.8 (C-1); 136.9 (C-1a); 145.2 (C-5a); 147.5 (C-4a); 147.6 (C-4); 149.1 (C-5).

Vladinol $\mathrm{F}$ was obtained as a colorless amorphous solid having a melting point of $105^{0}-107^{0}$. Its molecular formula was determined as $\mathrm{C}_{20} \mathrm{H}_{24} \mathrm{O}_{6}$ by ESI-MS ([M-H] $]^{-}$ion at $\mathrm{m} / z$ 359.2). The UV (216, 230 , and $284 \mathrm{~nm}$ ) spectra of this compound were typical for a phenolic chromophore. The ${ }^{13} \mathrm{C}-\mathrm{NMR}$ (Nuclear Magnetic Resonance) (Table-1) and DEPT (Distortion less Enhancement by Polarization Transverse) $(90,135)$ spectra of the isolated compound revealed 20 carbon signals including two aromatic rings $\left(\delta_{\mathrm{C}} 110.6-149.1\right)$, one oxygenated methine group $\left(\delta_{\mathrm{C}} 88.9\right)$, one methine group $\left(\delta_{\mathrm{C}} 55.5\right)$, two methylene groups $\left(\left(\delta_{\mathrm{C}} 32.9,35.8\right)\right.$, two oxygenated methylene groups $\left(\left(\delta_{\mathrm{C}} 62.2,65.0\right)\right.$, and two methoxy groups $\left(\delta_{\mathrm{C}} 56.4,56.8\right)$. The ${ }^{1} \mathrm{H}-\mathrm{NMR}$ spectra indicated five protons of two aromatic regions, one was at $\delta_{\mathrm{H}} 6.82(1 \mathrm{H}, d d, J=8.1,1.9, \mathrm{H}-2), \delta_{\mathrm{H}} 6.76(1 \mathrm{H}, d, J=8.1, \mathrm{H}-3), \delta_{\mathrm{H}} 6.95(1 \mathrm{H}, d, J=1.9, \mathrm{H}-6)$, and another was $\delta_{\mathrm{H}} 6.72(1 \mathrm{H}, s, \mathrm{H}-2 \mathrm{a})$ and $\delta_{\mathrm{H}} 6,73(1 \mathrm{H}, s, \mathrm{H}-6 \mathrm{a})$. Two singlet signals of methoxy group were at $\delta_{\mathrm{H}} 3.81(3 \mathrm{H})$ and $\delta_{\mathrm{H}} 3.85(3 \mathrm{H})$. Furthermore, in the ${ }^{1} \mathrm{H}-\mathrm{NMR}$ spectra showed a structure of propanol side chain at $\delta_{\mathrm{H}} 2.63(2 \mathrm{H}, t, J=6.5, \mathrm{H}-7 \mathrm{a}), \delta_{\mathrm{H}} 1.87(2 \mathrm{H}, m, \mathrm{H}-8 \mathrm{a}), \delta_{\mathrm{H}} 3.57(2 \mathrm{H}, t, J=5.4, \mathrm{H}-9 \mathrm{a})$. Four hydrogen proton signals at $\delta_{\mathrm{H}} 5.49(1 \mathrm{H}, d, J=6.3, \mathrm{H}-7), \delta_{\mathrm{H}} 3.46(1 \mathrm{H}, q, \mathrm{H}-8) \delta_{\mathrm{H}} 3.75(1 \mathrm{H}, d d$, $\left.J=6.0,9.2, \mathrm{H}-9^{\prime}\right)$, and $\delta_{\mathrm{H}} 3.83\left(1 \mathrm{H}, d d, J=5.6,9.6, \mathrm{H}-9{ }^{\prime \prime}\right)$ were a $-\mathrm{CH}-\mathrm{CH}-\mathrm{CH}_{2}$ - a structural unit which formed a dihydrofuran ring. The $\mathrm{HMBC}$ spectrum of this compound showed correlations between $\mathrm{H}-$ 7/C-4a, H-7/C-3a, H-7/C-2, H-7/C-6, H-2/C-7, H-6/C-1, H-6/C-7, and H-6a/C-4a. These correlations indicated that dihydrofuran ring fused at $\mathrm{C}-3 \mathrm{a}$ and $\mathrm{C}-4 \mathrm{a}$ of the aromatic ring and a phenyl group was located at $\mathrm{C}-7$ of dihydrofuran ring. Long-range couplings determined the placement propanol group at position $\mathrm{C}-1 \mathrm{a}$ of the aromatic ring in the $\mathrm{HMBC}$ spectrum between $\mathrm{H}-2 \mathrm{a} / \mathrm{C}-7 \mathrm{a}$ and $\mathrm{H}-8 \mathrm{a} / \mathrm{C}-1 \mathrm{a}$. Based on the spectroscopic analysis, the isolated compound was established as vladinol F (Fig.-1), 7phenyldihydrobenzofuranpropanol-type neolignane. ${ }^{12,13}$ We compared it with the previously reported Vladinol F (see Table-1) to validate the isolated compound.

Furthermore, the isolated compound was checked out as antiplasmodial. Methods previously described by Widyawaruyanti determined the antiplasmodial activity of the isolated compound. ${ }^{14}$ The samples were dissolved in DMSO and kept at $-20^{\circ} \mathrm{C}$ until use. The parasite $P$. falciparum (3D7) clone was propagated in a 24-well culture plate in the presence of $100,10,1,0.1$ and $0.01 \mu \mathrm{g} / \mathrm{ml} \mathrm{range} \mathrm{of}$ concentrations of the sample. Parasitaemia was monitored over 48 hours by making a blood smear fixed with $\mathrm{MeOH}$ and stained with Geimsa (Merck). The antiplasmodial activity test of the vladinol $\mathrm{F}$ toward $P$. falciparum showed the $\mathrm{IC}_{50}$ value of $3.51 \mu \mathrm{g} / \mathrm{ml}$. This result showed that vladinol $\mathrm{F}$ was good as an antiplasmodial agent. ${ }^{15}$
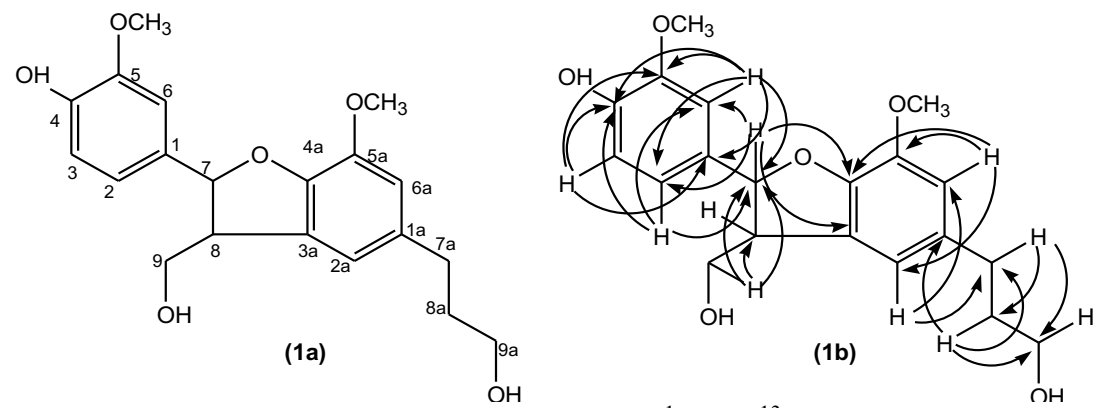

Fig.-1: (1a) Structure and (1b) HMBC Correlations $\left({ }^{1} \mathrm{H}<=>{ }^{13} \mathrm{C}\right)$ of the isolated compound 
RASĀYAN J. Chem.

Vol. 14 | No. 1 |161-165| January - March | 2021

Table-1: ${ }^{1} \mathrm{H}-\mathrm{NMR}(500 \mathrm{Mhz})$ and ${ }^{13} \mathrm{C}-\mathrm{NMR}(125 \mathrm{MHz})$ Spectral Data of Isolated Compound in methanol-d4 (Figure 3 \& 4, supplementary data) and Vladinol F (Isao Kouno, 1993) ${ }^{12}$

\begin{tabular}{|c|c|c|c|c|c|}
\hline \multirow[t]{2}{*}{$\mathrm{C}$} & \multicolumn{3}{|c|}{ Isolated Compound } & \multicolumn{2}{|c|}{$\begin{array}{c}\text { Vladinol F } \\
(\text { Isao Kouno, 1993) }\end{array}$} \\
\hline & $\begin{array}{l}\delta_{\mathrm{H}}(\mathrm{ppm}) \\
\text { (multiplet, } \\
J \text { in Hz) } \\
\end{array}$ & $\delta_{\mathrm{C}}(\mathrm{ppm})$ & $\begin{array}{l}\text { HMBC } \\
\left({ }^{1} \mathrm{H}<=>{ }^{13} \mathrm{C}\right)\end{array}$ & $\begin{array}{l}\delta_{\mathrm{H}}(\mathrm{ppm}) \\
\text { multiplet } \\
J \text { in Hz) }\end{array}$ & $\begin{array}{l}\delta_{\mathrm{C}} \\
(\mathrm{ppm})\end{array}$ \\
\hline 1 & - & 134.8 & - & - & 137.9 \\
\hline 2 & $\begin{array}{l}6.82(d d, 8.1 \& \\
1.9)\end{array}$ & 119.7 & $\mathrm{C}-4, \mathrm{C}-6, \mathrm{C}-7$ & $\begin{array}{l}6.93 \quad(d d, 8.1 \quad \& \\
1.8)\end{array}$ & 119.4 \\
\hline 3 & $6.76(d, 8.1)$ & 115.9 & $\mathrm{C}-1, \mathrm{C}-4, \mathrm{C}-5$ & $7.06(d, 8.1)$ & 118.9 \\
\hline 4 & - & 147.6 & - & - & 148.5 \\
\hline 5 & - & 149,1 & - & - & 152,1 \\
\hline 6 & $6.95(d, 1.9)$ & 110.6 & $\begin{array}{l}\text { C-1, C-2, C-4, } \\
\text { C-5, C-7 }\end{array}$ & $7.03(d, 1.8)$ & 111.3 \\
\hline 7 & $5.49(d, 6.3)$ & 88.9 & $\begin{array}{l}\text { C-2, C-6, C-8, } \\
\text { C-3a, C-4a }\end{array}$ & $5, .5(d, 5,9)$ & 88.6 \\
\hline 8 & $3.46(b r d q)$ & 55.5 & - & $\begin{array}{l}3.46(d d, 12.5 \& \\
5.5)\end{array}$ & 55.6 \\
\hline 9 & $\begin{array}{l}3.75 \& 3.83(d d \\
\& d d)\end{array}$ & 65.0 & C-7, C-8 & $3.73 \& 3.84(m)$ & 65.1 \\
\hline $1 \mathrm{a}$ & - & 136.9 & - & - & 137.0 \\
\hline $2 \mathrm{a}$ & $6.72(s)$ & 117.9 & C-6a, C-7a & $6.72(s)$ & 118.0 \\
\hline $3 a$ & - & 129.9 & - & - & 129.7 \\
\hline $4 \mathrm{a}$ & - & 147.5 & - & - & 147.5 \\
\hline $5 \mathrm{a}$ & - & 145.2 & - & - & 145.3 \\
\hline $6 a$ & $6.73(s)$ & 114.3 & $\begin{array}{l}\mathrm{C}-2 \mathrm{a}, \mathrm{C}-4 \mathrm{a}, \mathrm{C}- \\
5 \mathrm{a}\end{array}$ & $6.73(s)$ & 114.3 \\
\hline $7 \mathrm{a}$ & $2.63(t)$ & 32.9 & C-8a, C-9a & $2.62(t, 7.7)$ & 32.9 \\
\hline $8 \mathrm{a}$ & $1.87(p)$ & 35.8 & $\begin{array}{l}\text { C-1a, C-7a, C- } \\
9 a\end{array}$ & $1.82(\mathrm{~m})$ & 35.8 \\
\hline $9 \mathrm{a}$ & $3.57(t)$ & 62.2 & $C-7 a, C-8 a$ & $3.56(t, 6.4)$ & 62.3 \\
\hline $\begin{array}{l}5 \mathrm{a}-\mathrm{OCH}_{3} \\
8-\mathrm{OCH}_{3}\end{array}$ & $\begin{array}{l}3.81(s) \\
3.85(s) \\
\end{array}$ & $\begin{array}{r}56.4 \\
56.8 \\
\end{array}$ & - & $\begin{array}{l}3.82(s) \\
3.85(s) \\
\end{array}$ & $\begin{array}{r}56.5 \\
56.9 \\
\end{array}$ \\
\hline
\end{tabular}
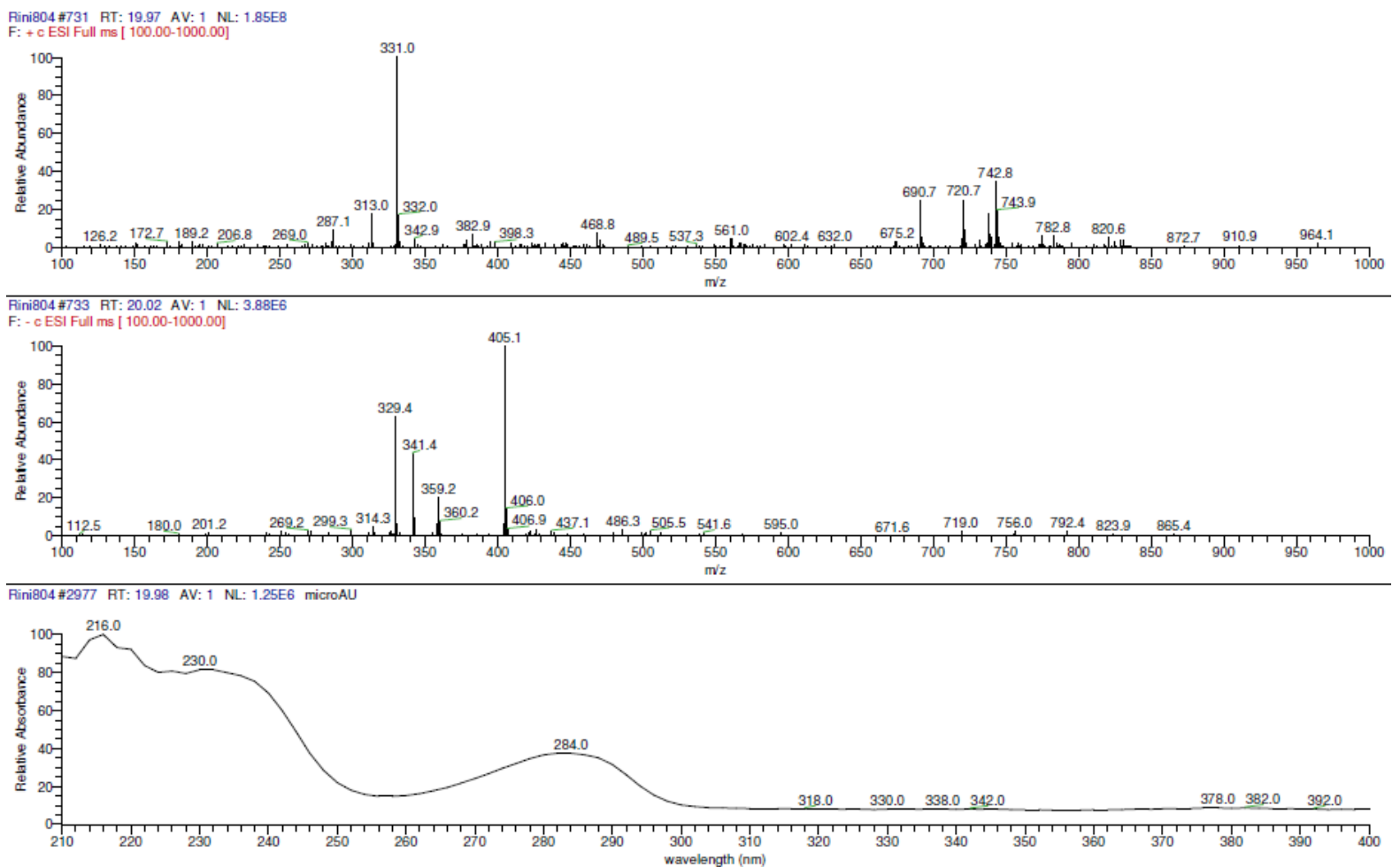

Fig.-2: ESI-MS Spectral Data of the Isolated Compound 


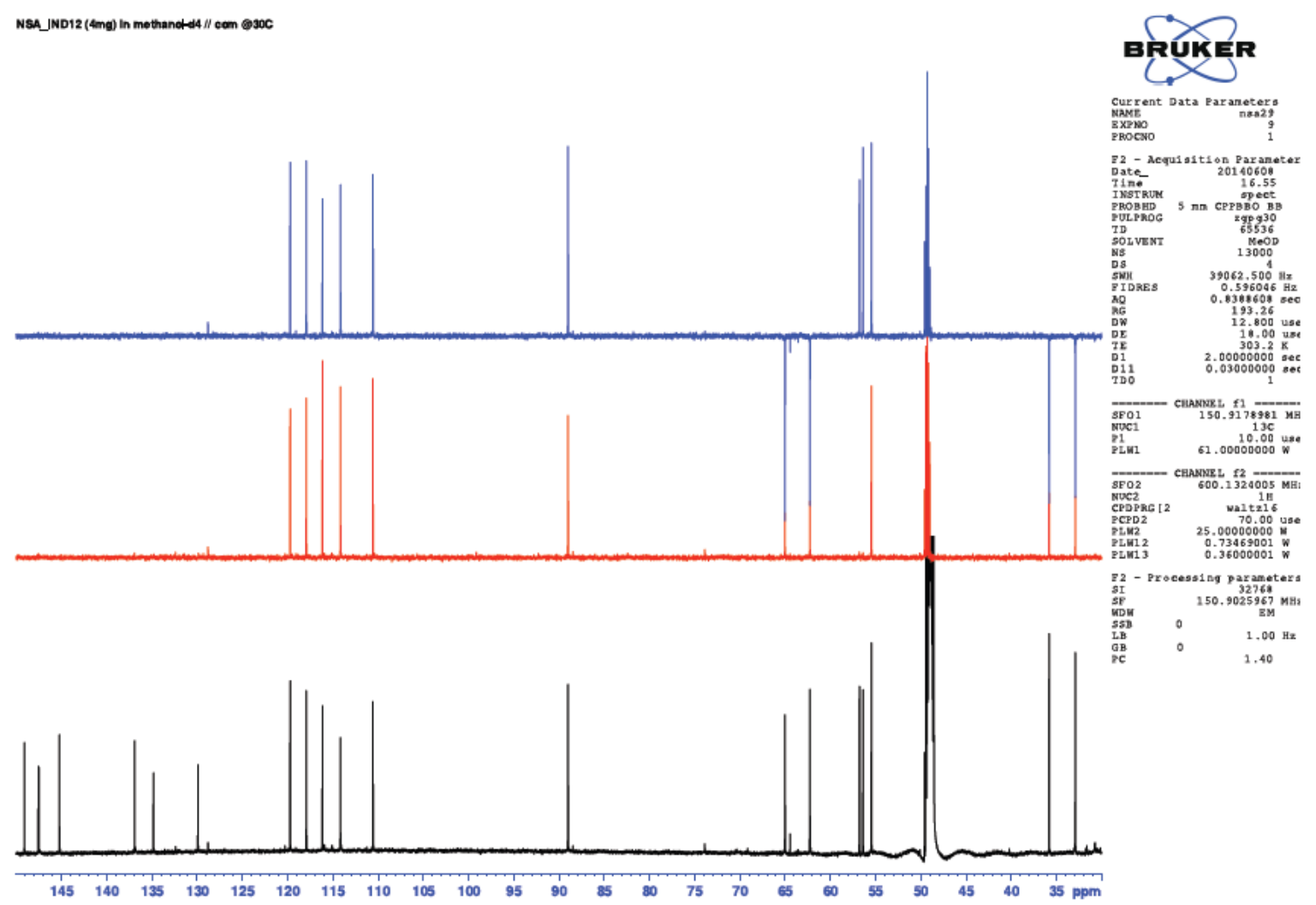

Fig. $-3{ }^{13} \mathrm{C}-\mathrm{NMR}$ and DEPT Spectral Data of the Isolated Compound

\section{CONCLUSION}

A neolignan, vladinol $\mathrm{F}$, was successfully isolated from the dissolved fraction in acetone-ether of Dryobalanops oblongifolia stem bark. The antiplasmodial activity test of the vladinol $\mathrm{F}$ toward $P$. falciparum showed the $\mathrm{IC}_{50}$ value of $3.51 \mu \mathrm{g} / \mathrm{mL}$. This result showed that vladinol $\mathrm{F}$ was good as an antiplasmodial agent.

\section{ACKNOWLEDGMENT}

We would like to thank: (1) Directorate General of Higher Education of Indonesia for financial supporting of this research and (2) Faculty of Pharmacy, Meijo University, Nagoya, Japan for measuring NMR and MS data.

\section{REFERENCES}

1. D. Kesuma1 , A.L. Nasyanka, M. Rudyanto, Siswandono, B.T. Purwanto, And I.G.A. Sumartha, Rasayan Journal of Chemistry, 13(3), 1914(2020), DOI:10.31788/RJC.2020.1335694

2. D. Handayani, R. Amilia, I. Aminah, T. Hertiani, N. P. Ariantari, and P. Proksch, Rasayan Journal of Chemistry, 13(3), 1817(2020), DOI:10.31788/RJC.2020.1335549

3. G. Swapna, D. Rakesh, and M. Estari, Rasayan Journal of Chemistry, 13(4), 4 (2020), DOI:10.31788/RJC.2020.1345889

4. WHO, World Malaria Report (2015), www.who.int/malaria

5. Purwaningsih, Ecological Distribution of Dipterocarpaceae Species in Indonesia, Biodeversitas, 5, 2 (2004), DOI: 10.13057/biodiv/d050210

6. M.F. Newman, P.F. Burges, T.C. Whitmore, Manual of Dipterocarps Series (Sumatra, Kalimantan, Jawa to Nuigini), Prosea-Indonesia, Bogor, pp.5-45, (1999)

7. N.S. Aminah, Y.M. Syah, E.H. Hakim, N. Aimi, M. Kitajima, H. Takayama, S.A. Achmad, Phytochemistry, 63 (2003), DOI:10.1016/s0031-9422(03)00274-7

8. N.S. Aminah, Y.M. Syah, E.H. Hakim, N. Aimi, S.A. Achmad, M. Niwa, Indonesia Journal Chemistry, 6(1)(2006), DOI:10.22146/ijc.21778

9. A. Wibowo, N. Ahmat, A.S. Hamzah, A.L.M. Low, S.A.S. Mohamad, H.Y. Khong, A.S. Sufian, N. Manshoor, H. Takayama, Fitoterapia, 83, 8 (2012), DOI:10.1016/j.fitote.2012.09.004

10. A. Wibowo, N. Ahmat, A.S. Hamzah, A.S. Sufian, N.H. Ismail, R. Ahmad, F.M. Jaafar, H. Takayama, Fitoterapia, 82, 4 (2011), DOI:10.1016/j.fitote.2011.02.006

11. Indriani, Yoshiaki Takaya, N.N.T. Puspaningsih, N.S. Aminah, Chemistry of Natural Compound, 53, 31 (2017), DOI:10.1007/s10600-017-2049-5 
12. Isao Kouno, Y. Yukari, S. Satomi, S. Miki, I. Yumi, and Y. Chun-Shu, Phytochemistry, 32, 6 (1993), DOI:10.1016/0031-9422(93)85182-Q

13. Qiu-Fen HU, Huai-Xue MU, Hai-Tao HUANG, Hua-Ying LV, Shu-Li LI, Pen-Fei TU, and GanPeng LI, Chemical and Pharmaceutical Bulletin, 59,11 (2011), DOI:10.1248/cpb.59.1421

14. Widyawaruyanti, S.K. Subehan, Kalauni, S. Awale, M. Nindatu. N.C. Zaini, D. Syafruddin, P.B. Setia Asih, Y. Tezuka, and S. Kadota, Journal of Natural Medicine, 61, 410(2007), DOI: 10.1007/s11418-007-0153-8

15. Ronan, J.S.J. Ademir, and B.O. Alaíde, Molecules, 14(8), 3037(2009), DOI:10.3390/molecules14083037

[RJC-6017/2020] 